\title{
Particle dynamics in a symmetrically driven underdamped inhomogeneous periodic potential system
}

\author{
D. Kharkongor ${ }^{1}$, W.L. Reenbohn ${ }^{2}$ and Mangal C. Mahato ${ }^{1} *$ \\ ${ }^{1}$ Department of Physics, North-Eastern Hill University, Shillong-793022, India and \\ ${ }^{2}$ Department of Physics, National Institute of Technology Meghalaya, Shillong-793003, India
}

(Dated: September 1, 2018)

\begin{abstract}
We numerically solve the underdamped Langevin equation to obtain the trajectories of a particle in a sinusoidal potential driven by a temporally sinusoidal force in a medium with coefficient of friction periodic in space as the potential but with a phase difference. With the appropriate choice of system parameters, like the mean friction coefficient and the period of the applied field, only two kinds of periodic trajectories are obtained for all possible initial conditions at low noise strengths: one with a large amplitude and a large phase lag with respect to the applied field and the other with a small amplitude and a small phase lag. Thus, the periodic potential system is effectively mapped dynamically into a bistable system. Though the directional asymmetry, brought about only by the frictional inhomogeneity, is weak we find both the phenomena of stochastic resonance, with ready explanation in terms of the two dynamical states of trajectories, and ratchet effect simultaneously in the same parameter space. We analyse the results in detail attempting to find plausible explanations for each.

PACS numbers: : 05.10.Gg, 05.40.-a, 05.40.Jc, 05.60.Cd
\end{abstract}

*Electronic address: mangal@nehu.ac.in 


\section{INTRODUCTION}

In this work we explore the possibility whether an inhomogeneous underdamped sinusoidal potential[1] system can be an appropriate candidate for obtaining ratchet current [2 8 ] while simultaneously exhibiting stochastic resonance [9-14]. In other words, whether in an inhomogeneous system one can obtain an optimally improved response to a subthreshold external field as the temperature is raised (stochastic resonance) in addition to exhibiting net asymmetric transport (ratchet effect) in a sinusoidal potential without the application of an external bias or with the application of an external field that, on its own, on the average, adds up to equal impulse in opposing directions.

Stochastic resonance was shown earlier to occur in sinusoidal potentials [15-17] contrary to its absence having been stressed previously[18]. It follows from the realization that when driven at a suitably large frequency, an underdamped particle moving in the sinusoidal potential shows two kinds of response trajectories, one with a large amplitude (and also large phase lag with respect to the drive) and the other with a small amplitude (and small phase lag). These solutions are quite robust and have the status of dynamical states. The characteristics that fully establish the trajectories as dynamical states are discussed in detail in Ref.[15]. The motion (trajectory) of the particle can be in either of these two states at any given instant of time. This is especially clearly observable at lower temperatures before the occurrence of stochastic resonance. Therefore, dynamically the system can be considered as bistable. The transition between these dynamical states, as the temperature is raised, can provide an explanation for the occurrence of stochastic resonance in periodic potentials. As far as the phenomenon of stochastic resonance is concerned, the sinusoidal potential thus recedes into oblivion and the problem is recast into one in a bistable system. This is important, for it is in the bistable systems that stochastic resonance is commonly observed.

The two dynamical states and the transitions between them refer mostly to trajectories about the minimum within a period of the periodic potential. However, the statement, "stochastic resonance in a periodic potential", is meaningful only if the motion includes not only inter-(dynamical)state transitions present but also movements between various wells (periods) of the potential. In other words, the 'bistability' must be maintained whichever period of the sinusoidal potential the particle happens to occupy as time progresses so that the full sinusoidal potential, and not just one period of it, is found to be accessed. Given the 
criterion to be satisfied, the occurrence of stochastic resonance is discussed only in terms of the two dynamical states and any reference to the sinusoidal potential is conveniently kept silent. However, sometimes, the reference to the interwell transitions has to be alluded to in order to check the credibility of the claim of occurrence of stochastic resonance in periodic potentials. In fact, well before stochastic resonance occurs, numerous interwell transitions take place. As the temperature becomes much larger than the temperature at which stochastic resonance occurs the transitions become so frequent that the two dynamical states become hardly discernible with separate identity, for the intervals of existence of the two states become too short (could even be less than a period).

By an inhomogeneous system we mean, in the present work, a system with nonuniform (space-dependent) friction coefficient. It has been theoretically shown earlier that an overdamped particle experiences a net effective driving force when subjected to a drift in a sinusoidal potential and also a sinusoidally modulated space-dependent diffusion, with the same period as the potential[19]. However, diffusion coefficient $D$ depends on both, the temperature $T$ and the friction coefficient $\gamma: D \sim \frac{T}{\gamma}$. The effect of temperature variation is considered dominant as it determines the local stability of states [19, 20]. It has been shown analytically that in an underdamped periodic potential a similarly periodic variation of temperature but with a phase difference leads to ratchet current[21]. This remarkable result was also supported numerically in a related work[22]. The effect of friction coefficient variation is weak and, unlike temperature variation, a mere similar periodic variation of friction coefficient is not expected to yield ratchet current; to yield ratchet current, in this case, one needs to apply, in addition, a zero-mean external forcing[23].

The ratchet effect has been studied in nonuniform friction periodic potential systems earlier too but mostly in the overdamped limit[24] unlike in the present case of underdamped systems. Also, the period of the drive taken earlier used to be very large, typically of the order of mean first-passage-time across a potential barrier. In these circumstances, stochastic resonance in the periodic potential systems was not found to occur and hence there was no question of exploring whether both the effects occur simultaneously in the same parameter space.

As stated earlier, in addition to the ratchet effect discussed above, the present work endeavours to observe SR simultaneously in the same parameter space. Though the present work is not experimental, it is not hard to think of an easy-to-visualize experimental situ- 
ation illustrating the present case. Consider a series of nodes and antinodes, created by a stationary pressure wave, representing sites of alternate low and high density of the medium. A particle, therefore, while moving along longitudinally, experiences a periodic variation of friction. In addition, if the particle is charged and an alternating electric potential field, of same wavelength as the pressure wave but with a phase difference, is created the particle will experience a spatially periodic force field. The application of a temporally sinusoidal external electric field completes the illustrative experimental situation to describe the problem at hand and allows the following questions to be asked. Is it possible to obtain a net (ratchet) current? If a thermal noise is added and increased gradually, either through the external field or the system itself, will the response of a host of particles to the external field be sharper or more smeared? In other words, does the quality of response, as conventionally measured by the (output) signal to (input) noise ratio, improve as the noise level is gradually increased? If the response shows a peak (stochastic resonance) at an intermediate temperature (noise strength) does the net particle current also exhibit a maximum, simultaneously[25]? These are some of the questions that will be attempted to be clarified in a model system.

These questions together have already been discussed earlier, however, in a uniform friction medium and subjected to a biharmonic external field[26], $F(t)=F_{0}(A \cos (\omega t)+$ $B \cos (2 \omega t))$. In the present work, the potential is considered sinusoidal $V(x)=-V_{0} \sin (x)$ and an external field of $F(t)=F_{0} \cos (\omega t)$. The asymmetry in the system is introduced via the phase shifted nonuniform friction $\gamma(x)=\gamma_{0}(1-\lambda \sin (x+\theta))$, with $0 \leq \lambda \leq 1$. In the case of temperature inhomogeneity, similar in form to the frictional inhomogeneity considered here, it has been shown earlier[21, 22] that the ratchet current $\bar{v}$ depends on the phase difference $\theta$ between the potential function and the temperature function as $\bar{v} \sim \sin (\theta)$. Thus, understandably, the ratchet current is maximum when the asymmetry is maximum, namely, for $\theta=\frac{\pi}{2}$ and the same should be true in the present case of frictional inhomogeneity as well.

Interestingly, for $\theta=\frac{\pi}{2}$, the inhomogeneous friction yields particle trajectories (and stochastic resonance) qualitatively similar to the uniform friction case in response to the same sinusoidal external field (see Sec. III). However, the inhomogeneous friction, in addition, helps in obtaining a net (ratchet) current. Since, in both the cases, the trajectories show same periodicity as the external field, the particle spends almost the same time in the small and large friction regions which lie, respectively, on either side of the potential minimum. 
The present situation is therefore quite different from the adiabatic drive case wherein the particle is likely to spend unequal durations in the two friction regions. Hence the reason put forth for the explanation of occurrence of ratchet current in the adiabatic limit will not hold in the finite frequency drive case[23]. A closer statistical analysis of the parts of trajectories in the two separate regions, however, hints at why the net (asymmetric) particle current should be expected.

The turning points of the trajectories on either side of potential minimum $\left(x=\frac{\pi}{2}\right)$ show interesting distributions. At very low temperatures, $(T<0.005)$, the mean distance of turning points, $d x_{r}$ (in the high friction side of the potential peak) and $d x_{l}$ (in the low friction side) from $x=\frac{\pi}{2}$ and their standard deviations, $\Delta x_{r}$ and $\Delta x_{l}$, about the mean values on both sides are same, showing that the motion remains symmetrical on both

directions, for all $\tau=\frac{2 \pi}{\omega}$. However, as $T$ is gradually increased the differences show up for $\lambda \neq 0$ and $\theta=\frac{\pi}{2}$. For $\tau=7.0$ and at low temperatures only large amplitude trajectories appear (see Sec. III, Fig. 9). In this case, one obtains $d x_{l}>d x_{r}$ and $\Delta x_{l}>\Delta x_{r}$ upto a reasonably large temperature. And, for $\tau=13.0$ and at low temperatures only small amplitude trajectories appear in which case one finds $d x_{r}>d x_{l}$ and $\Delta x_{l} \sim \Delta x_{r}$. However, since inter-well transitions and hence ratchet current is dominantly determined by the large amplitude trajectories, there is more likelyhood of right-to-left net transport than the other way round. Of course, differences of $d x_{l}$ and $d x_{r}$, etc. are very small (less than one percent) and the interwell transtions are thus decided by the rare large amplitude events.

In the next section II, the model system of our study will be described. In section III the numerical results based on our investigations will be presented in detail and in the last section IV our results will be discussed and summarised.

\section{THE MODEL}

We consider the motion of an ensemble of under-damped non-interacting Brownian particles each of mass $m$ in a periodic potential $V(x)=-V_{0} \sin (k x)$. The medium in which the particle moves is taken to be inhomogeneous in the sense that it offers a spatially varying friction with coefficient

$$
\gamma(x)=\gamma_{0}(1-\lambda \sin (k x+\theta))
$$


that leads/lags the potential by a phase difference $\theta$. This choice of friction coefficient breaks the right-left spatial symmetry of the system. Here, $\lambda$ is the inhomogeneity parameter, with $0 \leq \lambda \leq 1$ and hence $\gamma_{0}(1-\lambda) \leq \gamma(x) \leq \gamma_{0}(1+\lambda)$.

In addition, the potential is rocked by a sub-threshold periodic time-dependent forcing $F(t)=F_{0} \cos (\omega t)$, with $\omega=2 \pi / \tau$ as the rocking frequency and $\tau$ as the rocking period. The equation of motion of the particle subjected to a thermal Gaussian white noise $\xi(t)$ at temperature $T$ is given by the Langevin equation[27, 28],

$$
m \frac{d^{2} x}{d t^{2}}=-\gamma(x) \frac{d x}{d t}-\frac{\partial V(x)}{\partial x}+F(t)+\sqrt{\gamma(x) T} \xi(t) .
$$

with

$$
<\xi(t)>=0,<\xi(t) \xi\left(t^{\prime}\right)>=2 \delta\left(t-t^{\prime}\right)
$$

Here, and throughout the text, $<. .>$ correspond to ensemble averages.

For simplicity and convenience the equation is transformed in to dimensionless units [29] by setting $m=1, V_{0}=1, k=1$, with reduced variables denoted again by the same symbols. Thus, the Langevin equation takes the form

$$
\frac{d^{2} x}{d t^{2}}=-\gamma(x) \frac{d x}{d t}-\frac{\partial V(x)}{\partial x}+F(t)+\sqrt{\gamma(x) T} \xi(t)
$$

where the potential is reduced to $V(x)=-\sin (x)$ and

$$
\gamma(x)=\gamma_{0}(1-\lambda \sin (x+\theta))
$$

Equation (2.4) is numerically solved (i.e., integrated using Ito definition) to obtain the trajectories $x(t)$ of the particle for various initial conditions 30 32]. For initial positions

$x(t=0)$ the period $-\frac{\pi}{2}<x \leq \frac{3 \pi}{2}$ is divided uniformly into either $n=100$ or $n=200$ parts (and hence $n$ initial positions)and the initial velocity $v(t=0)$ is set equal to zero throughout in the following.

\section{NUMERICAL RESULTS}

For each trajectory, corresponding to one initial position $x(0)$, the work done by the field $F(t)$ on the system, or the input energy, is calculated as [33]:

$$
W(0, N \tau)=\int_{0}^{N \tau} \frac{\partial U(x(t), t)}{\partial t} d t
$$


where $N$ is a large integer denoting the number of periods taken to reach the final point of the trajectory. The effective potential $U$ is given by

$$
U(x(t), t)=V(x(t))-x(t) F(t) .
$$

The mean input energy per period, for the particular trajectory, is therefore given by

$$
\bar{W}=\frac{1}{N} W(0, N \tau)
$$

For the deterministic case $\left(T \rightarrow 0\right.$, for example $\left.T=10^{-6}\right)$ and also at low temperatures (low noise strengths, typically less than about $10 \%$ of the largest potential barrier corresponding to $F(t))$ the trajectories $x(t)$ appear to be periodic and similar in nature to that of $F(t)$. Since $V(x)$ is not explicitly dependent on time, all of the contribution to $W(0, N \tau)$ comes from the second term in $U$, Eq. (3.2), because of the phase difference between $F(t)$ and $x(t)$. The nonzero phase difference also implies hysteresis $\overline{x(F)}$, whose area is a measure of energy dissipation per period by the system. A typical mean hysteresis loop is shown in Fig.1. Not surprisingly, therefore, it turns out that the corresponding mean hysteresis loop area $\bar{A}$ is same as $\bar{W}$. The overall mean input energy per period, $\langle\bar{W}\rangle$, is calculated as an (ensemble) average over all the $n$ trajectories (corresponding to all the initial positions considered). Again, we find that $\langle\bar{A}\rangle=\langle\bar{W}\rangle$. Similarly, the mean velocity $\bar{v}$, over one trajectory, is calculated as

$$
\bar{v}=\frac{1}{N \tau} x(t=N \tau),
$$

and the overall mean (net) velocity or the ratchet current, $\langle\bar{v}\rangle$, is calculated as the ensemble average over all the $n$ trajectories. Typically, as discussed below, $N=200000$ for small temperatures and $N=500000$ for large temperatures. The value of $N$ is chosen to have relatively small error bars so that the qualitative features of the results (see below) are not obscured.

The error bars are calculated assuming the numerical procedure adopted to be correct and errors occurring only due inherently to the stochastic dynamics of the system. We calculate the $x(N \tau)$ for each initial condition used to calculate $\bar{v}=\frac{x(N \tau)}{N \tau}$. The deviations of these $\bar{v}$ from the mean $\langle\bar{v}>$ calculated as an ensemble average over all trajectories with distinct initial conditions. The standard deviations $\Delta v$ form the error bars.

At large temperatures the effect of frictional inhomogeneity gets manifest. However, one needs to calculate the mean values carefully. The frictional inhomogeneity provides only a 


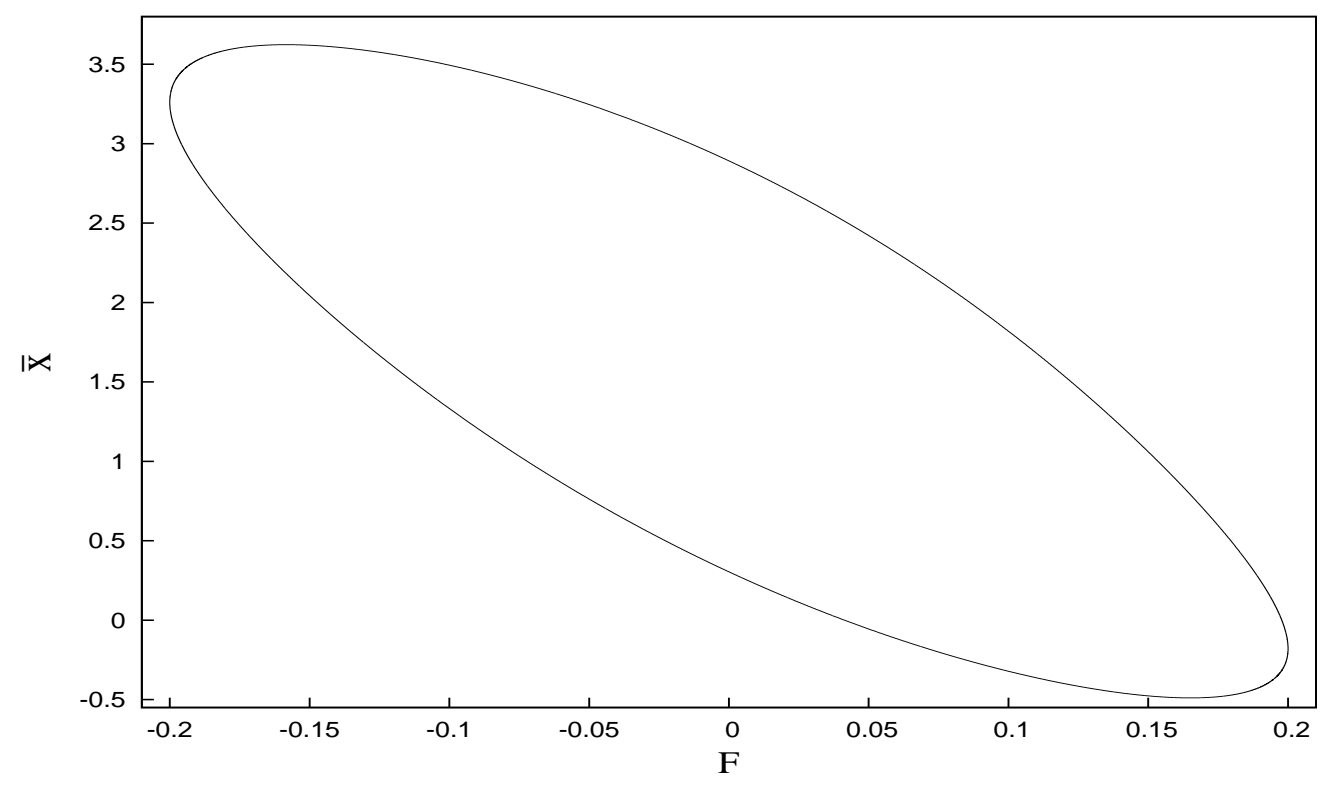

FIG. 1: Variation of $\bar{x}$ as a function of the forcing. Here, $\tau=8.0, \theta=0.5 \pi, \gamma_{0}=0.07, \lambda=0.9$, $T=0.000001$ and $F_{0}=0.2$. This loop represents a large amplitude state.

weak (left-right) asymmetry. As a consequence, the ratchet current $\langle\bar{v}\rangle$ obtained is not large and the standard deviations, $\Delta v$ (errorbars), are not small, though smaller than the mean $\langle\bar{v}\rangle$. This is especially true when the temperatures are large. Therefore, whereas for smaller temperatures $T<0.5$ one gets sensible $\left\langle\bar{v}>\right.$, values, say $\frac{\Delta v}{<\bar{v}>}<0.2$, when averaged over 200000 periods of the drive, it takes 500000 periods at temperatures $T>1.0$ to obtain similar sensible $\langle\bar{v}\rangle$ values. Figs. 2 and 3 illustrate the above statement succinctly.

In Fig. 3 is also plotted $\frac{2 \pi \Delta N}{N \tau}$ as a function of temperature. Here $\Delta N$ is the difference between the number of transitions to the left and right direction potential wells and $N$ is the total number of periods of $F(t)$ used to obtain $\Delta N$. The figure shows that $\left\langle\bar{v}>\right.$ and $\frac{2 \pi \Delta N}{N \tau}$ almost coincide. Note that the possibility of the particle making transition to a potential well farther than the adjacent well of the starting well before getting trapped is not ruled out, though such events are rare at lower temperatures but not so rare at higher temperatures. (These events are actually observed in the trajectory plots.) This naturally brings in the question of distribution of jump lengths (in terms of number of wells) on either directions and hence the dependence of net displacements (and ratchet currents) on the distributions. However, from the matching of $\left\langle\bar{v}>\right.$ and $\frac{2 \pi \Delta N}{N \tau}$ in Fig.3 shows that the disribution is sharply peaked at the jump length of $2 \pi$, or one well-length, on either directions; distributions are 


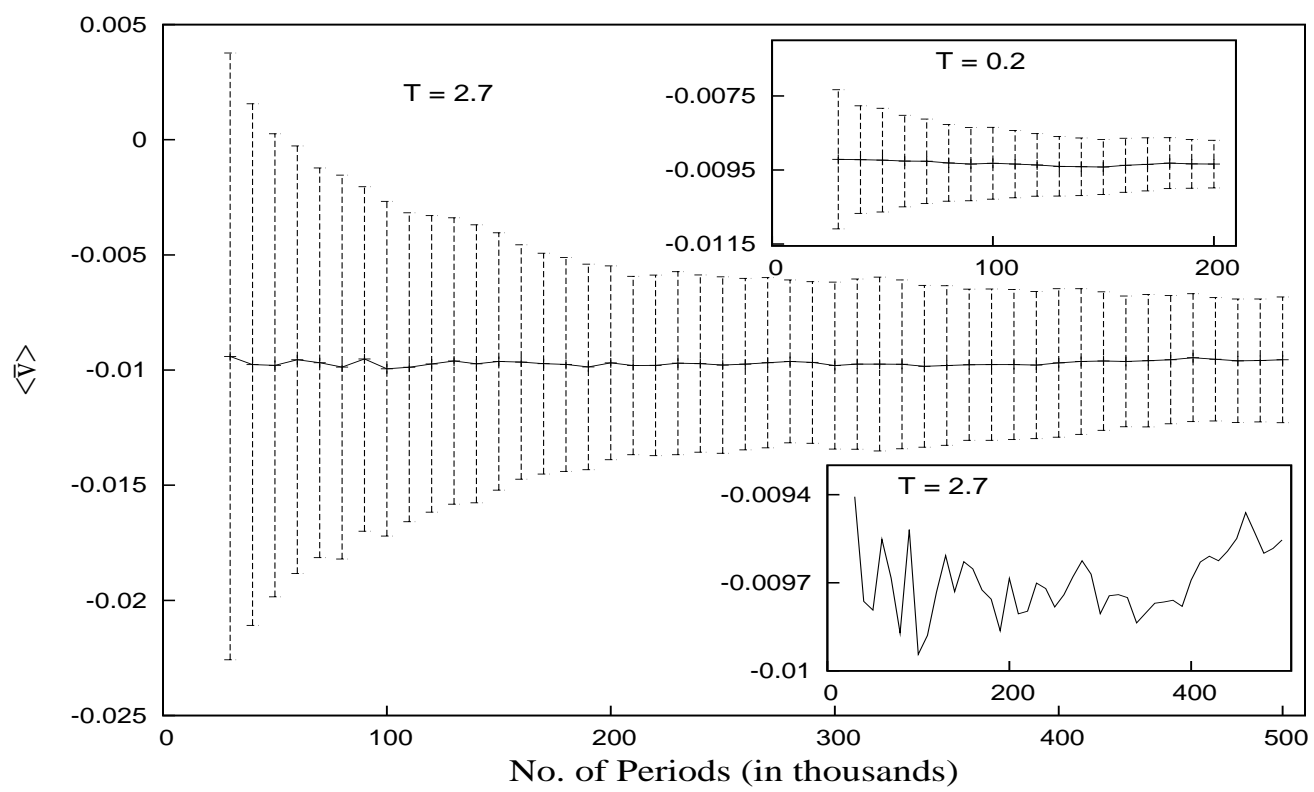

FIG. 2: Variation of $\langle\bar{v}>$ as a function of the number of periods of the forcing used to calculate $\bar{v}$ 's. The data for $<\bar{v}>$ had been recorded for every 10000 cycles and the corresponding error bars are calculated. Here, $\tau=7.7, \theta=0.5 \pi, \gamma_{0}=0.07, \lambda=0.9, T=2.7$ and $F_{0}=0.2$. The inset in the bottom-right shows the variation of $\langle\bar{v}>$ without error bars. The inset in the top-right corner is same as the main graph but for $T=0.2$. (Note: The labels on the axes of the insets are same as those of the main graph).

almost identical on both the directions. Of course, a finite small tail of the distributions corresponding to larger lengths, at least at higher temperatures, is always there.

Of course, clearly, the quantities of our interest $\langle\bar{W}\rangle$ and $\langle\bar{v}\rangle$ depend on the parameters $F_{0}$ and $\omega=\frac{2 \pi}{\tau}$ of the external field $F(t)$. In what follows we shall keep the amplitude $F_{0}$ fixed and equal to 0.2 throughout. The chosen value of $F_{0}$ is not only because it corresponds to a subthreshold forcing but it is in a range where one is expected to get desired sensible results[16].

\section{A. Dynamical states of trajectories at low temperatures}

Before we begin presenting the numerical results at finite temperatures, we examine the trajectories at a temperature close to zero $(T=0.000001)$ as a response to an external field $F(t)$. The nonuniformity of the friction coefficient $\gamma(x)=\gamma_{0}(1-\lambda \sin (x+\theta))$ is measured by 


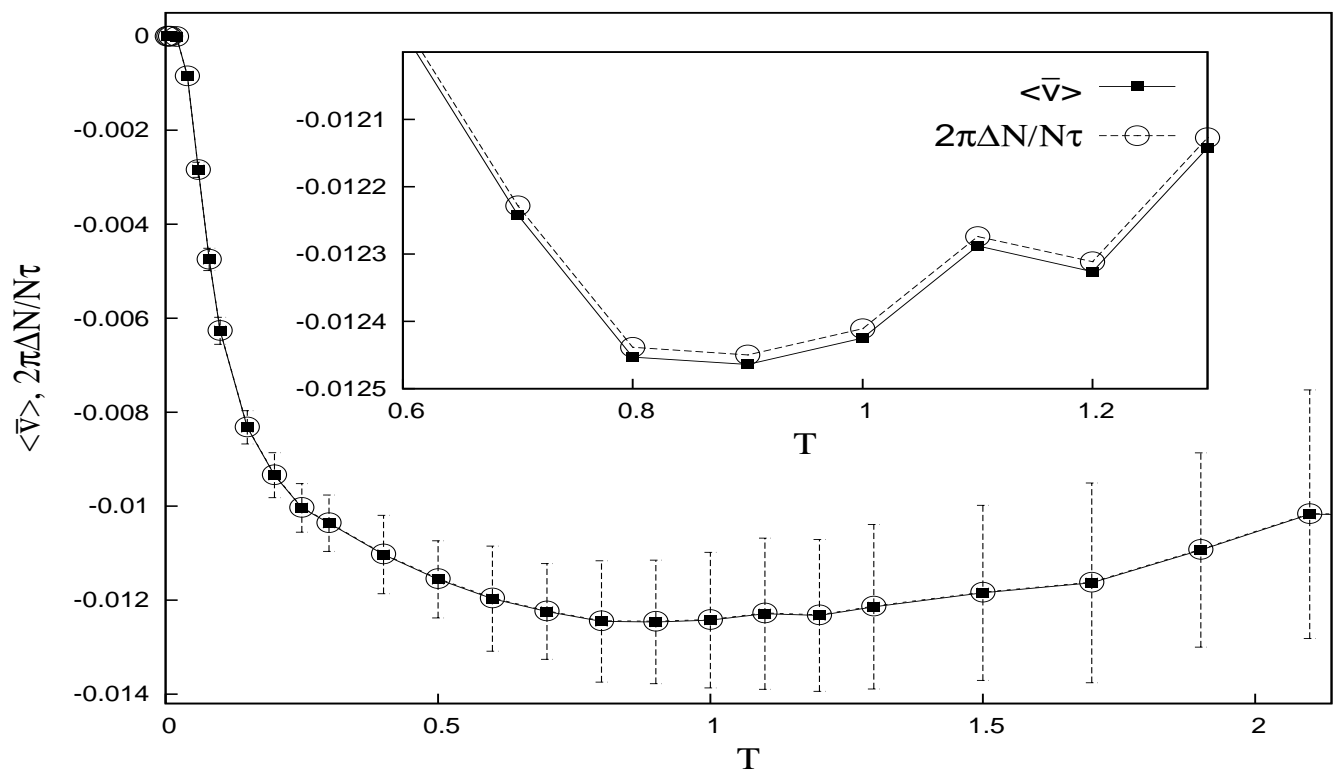

FIG. 3: Variation of $\langle\bar{v}\rangle$ as a function of $\mathrm{T}$ is shown (dark squares with errorbars) for $\tau=7.7$, $\theta=0.5 \pi, \gamma_{0}=0.07, \lambda=0.9$, and $F_{0}=0.2$. In the graph are also included the plot (open circles) of $\frac{2 \pi \Delta N}{N \tau}$, where $\Delta N$ is the difference between the number of left and right interwell hoppings, and $N$ is the corresponding total number of periods of $F(t)$ considered. The inset shows the magnified image of the main plot (without the errorbars) for a limited range of $\mathrm{T}$ values showing that both the quantities $\left\langle\bar{v}>\right.$ and $\frac{2 \pi \Delta N}{N \tau}$ are very close but not exactly equal.
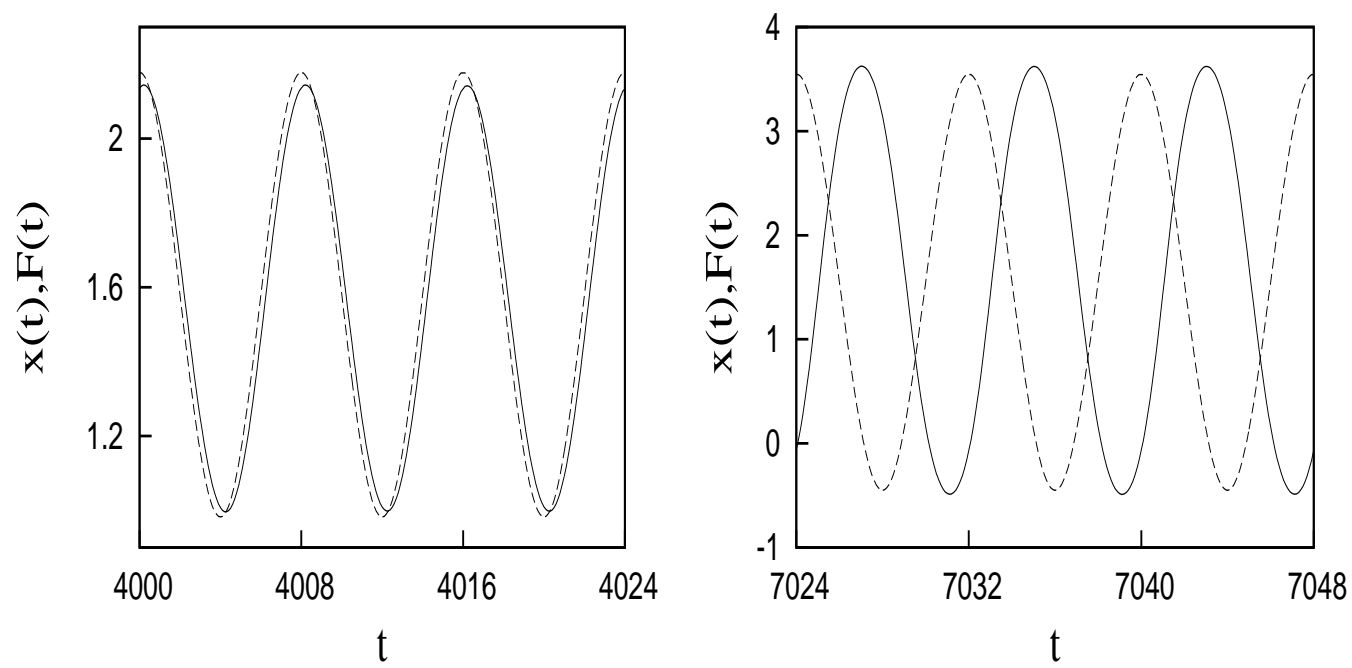

FIG. 4: The figure shows the two dynamical states of trajectories: SA state (left), with $\mathrm{x}(0)=$ $0.40 \pi$ and LA state (right), with $\mathrm{x}(0)=-0.35 \pi$, occuring when driven by a cosinusoid of same amplitude $F_{0}=0.2$ at $T=0.000001$, with $\lambda=0.9, \gamma_{0}=0.08, \theta=0.50 \pi$ and $\tau=8.0$. For both the figures, the dashed lines represent $F(t)$ multiplied by 2.99 centred at 1.58 and by 10 centred at 1.55 , respectively, for easy comparison. 
the value of $\lambda$ and its phase shift with respect to $V(x)=-\sin (x)$ by $\theta$. Fig.4 shows typical trajectories in the two states of large amplitude (LA) and small amplitude (SA) depending on the initial conditions chosen but for the same $F(t)$. These trajectories are periodic as $F(t)$ and are characterized by amplitude $x_{0}$ and phase lag (with respect to $F(t)$ ) $\phi$. Since, the dynamics being stochastic, the amplitude and phase lag are not the same in every period of $F(T)$, only their average values make sense. We measure them in either of the two ways: (i) we calculate the mean values of the quantities over several periods from the trajectories, or (ii) calculate from the mean hysteresis loops, Fig.1, considering this approximately to be an ellipse. However, the second method becomes impractical when the hysteresis loop becomes non-elliptic and in that case the first method is the only option.

In Figs.5, the variation of $x_{0}$ is shown as a function of $\lambda$ for various values of $\theta$ in the two states: LA state (a) and SA state (b). The percentages of variations are calculated with reference to values at $\lambda=0$. In Figs. $5(\mathrm{c})$ and $5(\mathrm{~d})$, the variations of $\phi$ are shown. It is to be noted, from the figures, that for $\theta=\frac{\pi}{2}$ both $x_{0}$ and $\phi$ almost remain constant (variation less than $0.03 \%$ ) over the entire range of $0<\lambda<1$. For $\theta<\frac{\pi}{2}, x_{0}$ increases as a function of $\lambda$ whereas for $\theta>\frac{\pi}{2}, x_{0}$ decreases with increase of $\lambda$ for both LA and SA states. However, these variations are very small, less than $2 \%$ at $\lambda=.9$. In the SA state the values of $\phi$ themselves are very small and hence their variations, though very small, appear to be large in percentage. In this state, for $\theta<\frac{\pi}{2}, \phi$ decreases and for $\theta>\frac{\pi}{2}$, it increases as a function of $\lambda$. The LA state has large values of $\phi$, for example, $\phi \sim 0.8 \pi$ at $\theta=\frac{\pi}{2}$. In this LA state, as $\lambda$ is increased from a small value $(\lambda \approx 0)$ to a large value, $\phi$ increases for $\theta<\frac{\pi}{2}$ whereas it decreases for $\theta>\frac{\pi}{2}$. These variations are comparatively large $(\leq 10 \%)$. Since the variations of $x_{0}$ are small the variations of the hysteresis $(\overline{x(F)})$ loop area $\bar{A}$ (or the input energy $\bar{W}$ ) in the two states are determined essentially by the variations of $\phi$. Note that $\phi=\frac{\pi}{2}$ should have the largest $\bar{W}$ for a given $x_{0}$. These varations of $\bar{W}$ are shown in Figs. 5(e) and 5(f).

In what follows we shall keep the value of $\lambda=0.9$ fixed. For $\lambda=0.9$ the results of Figs. 5 are summarized in Figs. $6(\mathrm{a}-\mathrm{f}): x_{0}(\theta), \phi(\theta)$, and $\bar{W}(\theta)$ for both LA and SA states. Again, the variation of $\bar{W}(\theta)$ is determined by the variation of $\phi(\theta)$; for the LA state $\phi$ decreases towards $\frac{\pi}{2}$ (and hence $\bar{W}$ increases), whereas for the SA state $\phi$ increases as $\theta$ is increased (again thereby increasing $\bar{W}$ ). Note that, since the particle moves in a periodic potential with a potential barrier of magnitude 2 whereas the drive amplitude is only 0.2 (and hence maximum reduction in potential barrier $=0.4 \pi$ ) the particle will remain confined to the 

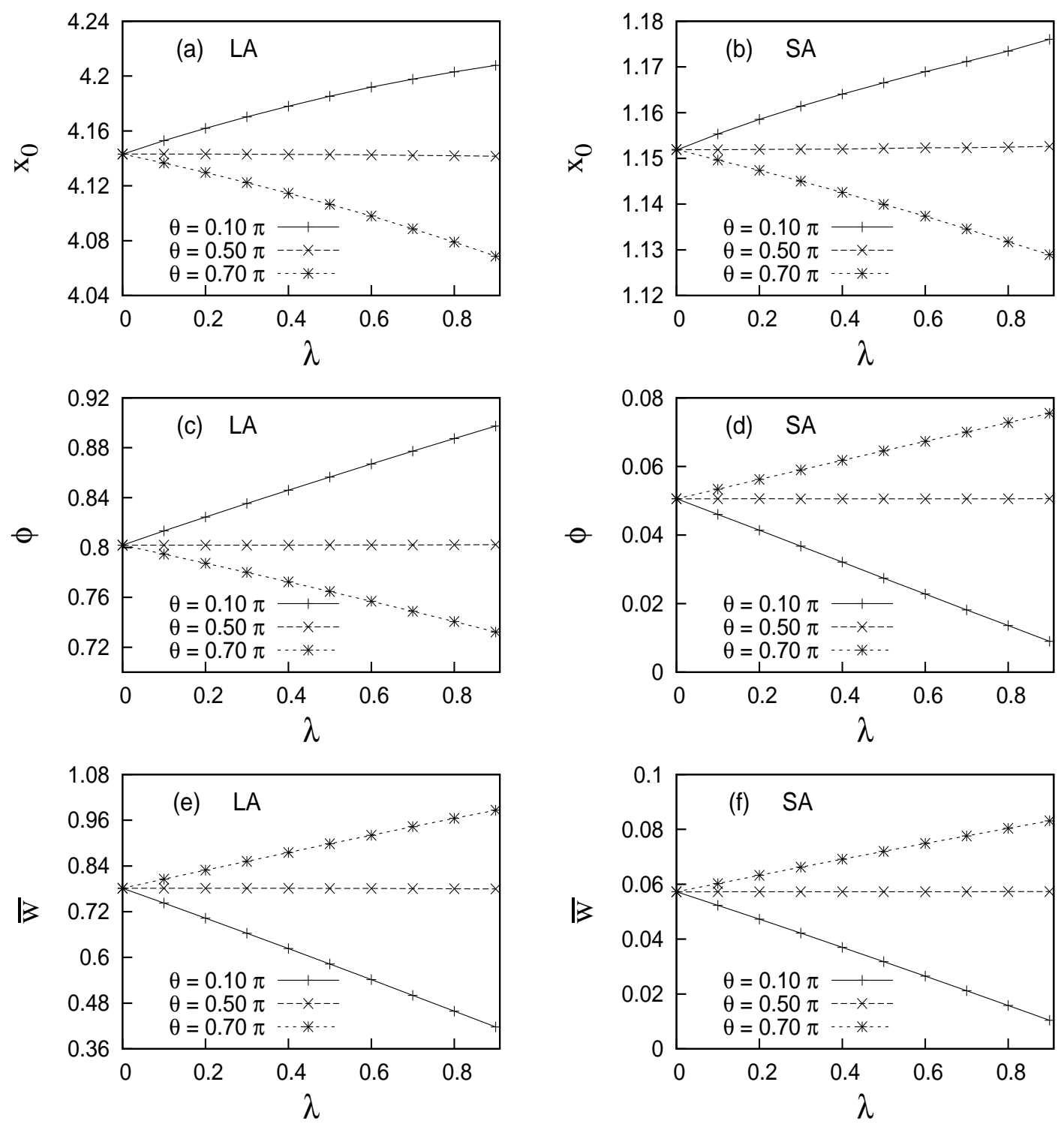

FIG. 5: The variations of $x_{0}, \phi$ and $\bar{W}$ as a function of $\lambda$ for both LA and SA states with $F_{0}=$ $0.2, \tau=8.0, \gamma_{0}=0.07$ at $T=0.000001$ and various values of $\theta$ as indicated.

same well of the potential at low temperatures. Only when the temperature is increased that the particle will have any chance of overcoming the potential barrier. Therefore, the mean net velocity $\langle\bar{v}>$ is zero at low temperatures even though $<\bar{W}>$ is nonzero at all temperatures.

Figure 7 shows the coexistence boundary of the two dynamical states in the $\left(\gamma_{0}, \tau\right)$ space for $\theta=0.25 \pi, 0.5 \pi$, and $0.75 \pi$. In the inset of the figure are shown the loci of various fractions of SA state within the coexistence boundary for $\theta=0.5 \pi$. Similar loci for other values of $\theta$ have also been calculated but are not shown here. However, the consequences of 

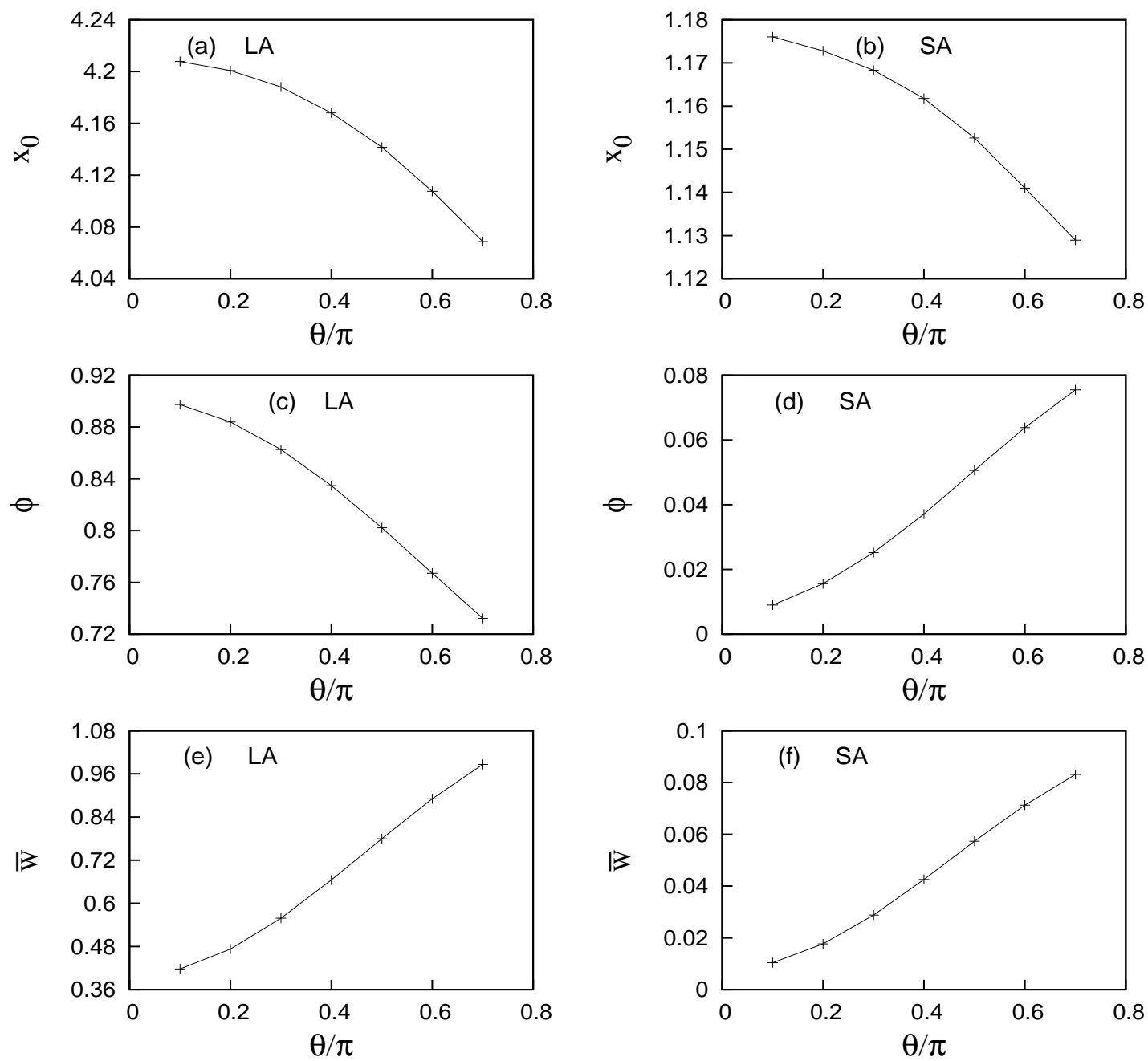

FIG. 6: The variations of $x_{0}, \phi$ and $\bar{W}$ as a function of $\theta$ for both LA and SA states with $F_{0}=$ $0.2, \tau=8.0, \gamma_{0}=0.07$ at $\mathrm{T}=0.000001$, keeping $\lambda=0.9$ fixed.

such fractions at any point in the $\left(\gamma_{0}, \tau\right)$ space will be discussed later. Clearly, the upper limit of $\gamma_{0}$ for the coexistence region decreases as $\theta$ increases. This behaviour of $\theta$ dependence can be understood if the relationship between the potential $V(x)$ and $\gamma(x)$ is examined. It will be easier to visualize if a rectangular profile of $\gamma(x)$ is considered, for illustration, instead of a sinusoidal profile. This is justified because it has been found that rectangular and sinusoidal profile of $\gamma(x)$ yield essentially similar transport characteristics.

In Fig.8, as an illustration, the potential $V(x)$ and rectangular $\gamma(x)$ are plotted together for the phase difference $\theta=0.5 \pi$ (a) and $\theta=0.25 \pi(\mathrm{b})$. Since $V(x)$ is sinusoidal the space between two consecutive minima forms a period. From Fig.8(a) one can see that $\gamma(x)$ is large over the entire half period to the left of $V(x)$ peak whereas $\gamma(x)$ is small over the rest 


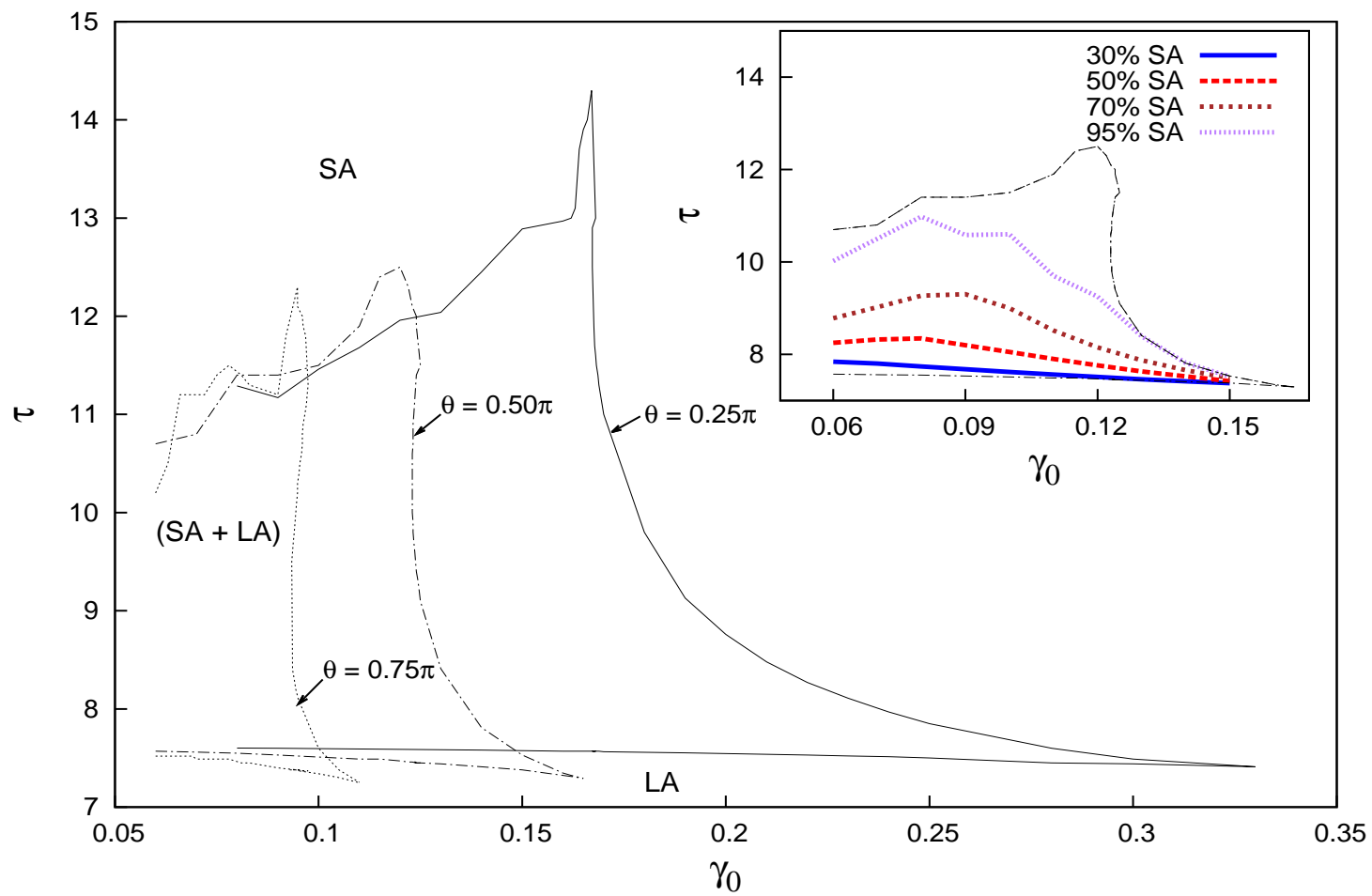

FIG. 7: The figure shows the boundaries of regions of coexistence of SA and LA states for $\theta=$ $0.25 \pi, 0.5 \pi$ and $0.75 \pi$ at $T=0.000001$. Observe that $\theta=0.25 \pi$ provides a larger maximum limiting value of $\gamma_{0}$ in the $\left(\tau, \gamma_{0}\right)$ space for the coexistence of the dynamical states. Clearly, with $\theta=0.75 \pi$, the limiting value of $\gamma_{0}$ has reduced significantly. In the inset are shown the loci of various percentages of populations of the SA state for $\theta=0.5 \pi$ at $\mathrm{T}=0.000001$.

half period, to the right, of $V(x)$ peak. Therefore, a particle will experience a moderate effective damping force, of magnitude, say $F_{\text {eff }}$, measured roughly by the averaged product $\gamma(x) v(x(t))$ over a period. On the other hand, from Fig.8(b), for $\theta=0.25 \pi$, one can see that at the bottom of the potential, where the particle velocity is expected to be large, the friction coefficient $\gamma$ is small and at the peak of the potential, where the velocity should be small the friction is large. Thus, one can conclude that the effective damping force experienced by the particle in this case will be smaller than $F_{\text {eff }}$ of the former case and hence the particle will be more mobile in this case of smaller $\theta$. From a similar consideration one can see that the effective damping force experienced by the particle in case of, say, $\theta=0.75 \pi$ will be larger than $F_{\text {eff }}$. In other words, the average mobility of the particle decreases as a function of $\theta$ for the same forcing and the potential function. Though difficult to define, one can think of a uniform effective friction coefficient $\overline{\gamma_{0}}$ for each $\theta$ (for nonuniform $\gamma(x)$ ) for a given 

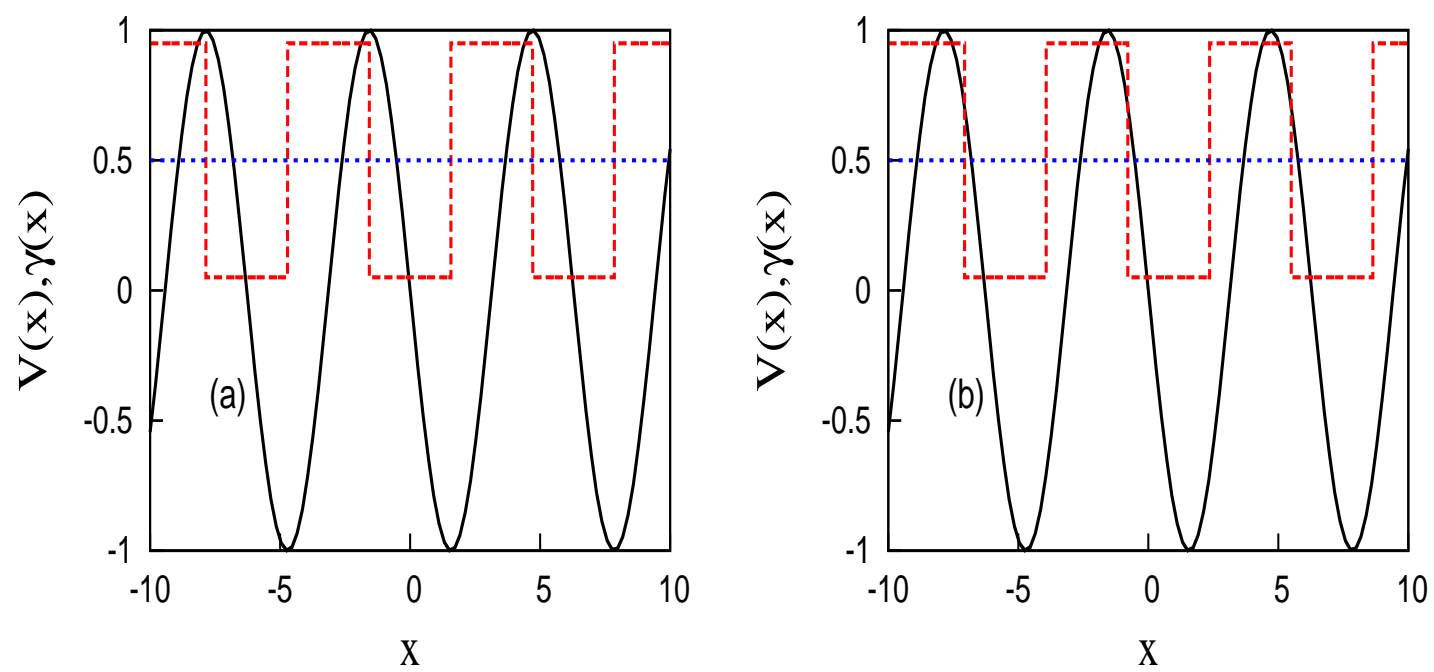

FIG. 8: The above two figures compare the the potential $\mathrm{V}(\mathrm{x})$ (black) and the (square-wave type) friction coefficient $\gamma(\mathrm{x})$ (red). The left figure is for $\theta=0.50 \pi$ and the right figure is for $\theta=0.25 \pi$. The blue line in both the figures is the mean value of $\gamma(\mathrm{x})$, here, taken as $\gamma_{0}=0.5$ for better comparison.

potential function and external field, as in case of ref.[15]. Thus, one can argue that the average effective friction coefficient, $\overline{\gamma_{0}}$ increases monotonically as $\theta$ is changed from 0 to $\pi$ in $\gamma(x)=\gamma_{0}(1-\lambda \sin (x+\theta))$. This explains why the $\left(\gamma_{0}, \tau\right)$ region of coexistence of the two dynamical states in Fig.7 shrinks as $\theta$ increases in order to have the same effective $\gamma$ at the upper coexistence limit.

\section{B. Ratchet current and input energy at finite temperatures}

From the above analysis one can conclude that for a given pair of $\left(\gamma_{0}, \tau\right)$ values the effective friction coefficient $\overline{\gamma_{0}}$ increases with $\theta$ and hence, as can be seen in Fig.9, the total number $\left(N_{t}\right)$ of interwell transitions at a given temperature $T$ decreases monotonically. This, however, does in no way imply that the ratchet current decreases monotonically with increasing $\theta$.

As can be seen, in Fig.10, the ratchet current, which is also a measure of difference between the interwell transitions in the right and left directions, peaks around $\theta=0.5 \pi$. Since $\theta=0.5 \pi$ provides the largest frictional asymmetry one would ideally think of the ratchet current to be maximum at $\theta=0.5 \pi$. However, at lower temperatures the ratchet 


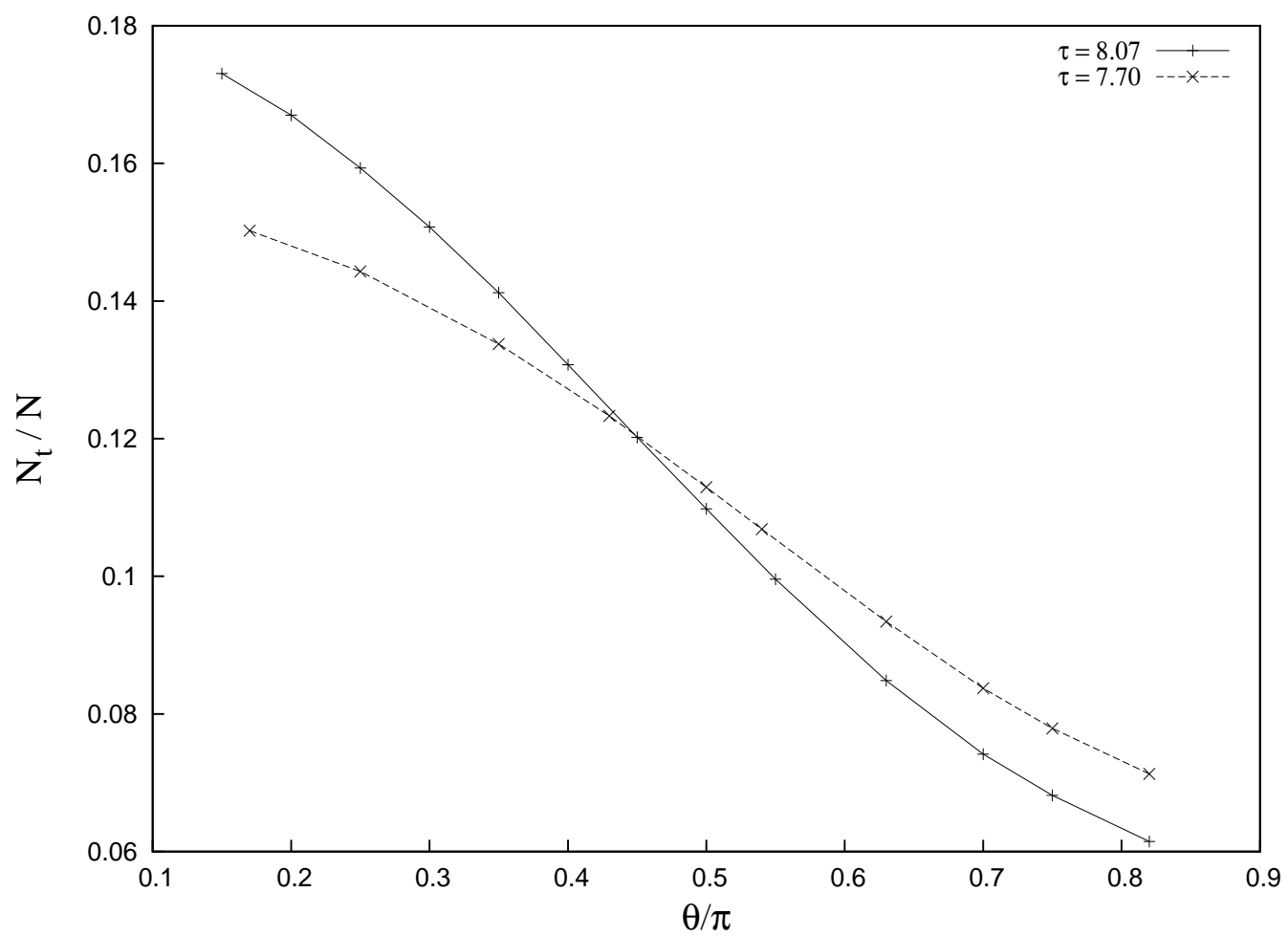

FIG. 9: The figure shows the decrease of the fraction of the total number of interwell hoppings $\left(\frac{N_{t}}{N}\right)$ as a function of $\theta$, for two $\tau$ values, $\tau=8.07$ (upper plot) and $\tau=7.70$ (bottom plot) in 200000 periods of $F(t)$. Here, $T=0.2, F_{0}=0.2, \gamma_{0}=0.07$, and $\lambda=0.9$.

current actually peaks at $\theta<0.5 \pi$, Fig.10. This is because lower $\theta$ corresponds to lower effective $\overline{\gamma_{0}}$ and hence being more mobile interwell transitions begin at lower temperatures than for larger $\theta$. Thus ratchet current grows faster, beginning at lower temperatures, for smaller $\theta$ and hence dominate before the frictional asymmetry overwhelms it at larger temperatures where one can find ratchet current to be the maximum for $\theta=0.5 \pi$, inset of Fig.10.

In Fig.11, the input energy $<\bar{W}>$ is plotted as a function of $\theta$ for two temperatures, (i) $T=0.000001$, based on the weightage average of results for LA and SA states (Figs. $6(\mathrm{e}, \mathrm{f})$ ), and (ii) $T=0.2$, for $\gamma_{0}=.07$ and $\tau=7.7$. At low temperatures $\langle\bar{W}\rangle(\theta)$ strongly depends on the choice of the point $\left(\gamma_{0}, \tau\right)$ and the variation can be explained with the help of the coexistence diagrams, Fig.7 and the loci of fractions of the SA states. In the inset of Fig. 11 are drawn some representative loci of the fractions of the SA state for $\theta=0.25 \pi, 0.5 \pi$, and $0.75 \pi$ together around the point $(0.07,7.7)$ in the $\left(\gamma_{0}, \tau\right)$ space. One can find that the fractions of the SA states for $\theta=0.75 \pi$ is $38.5 \%$, for $\theta=0.5 \pi$ it is $25 \%$ and for $\theta=0.25 \pi$ 


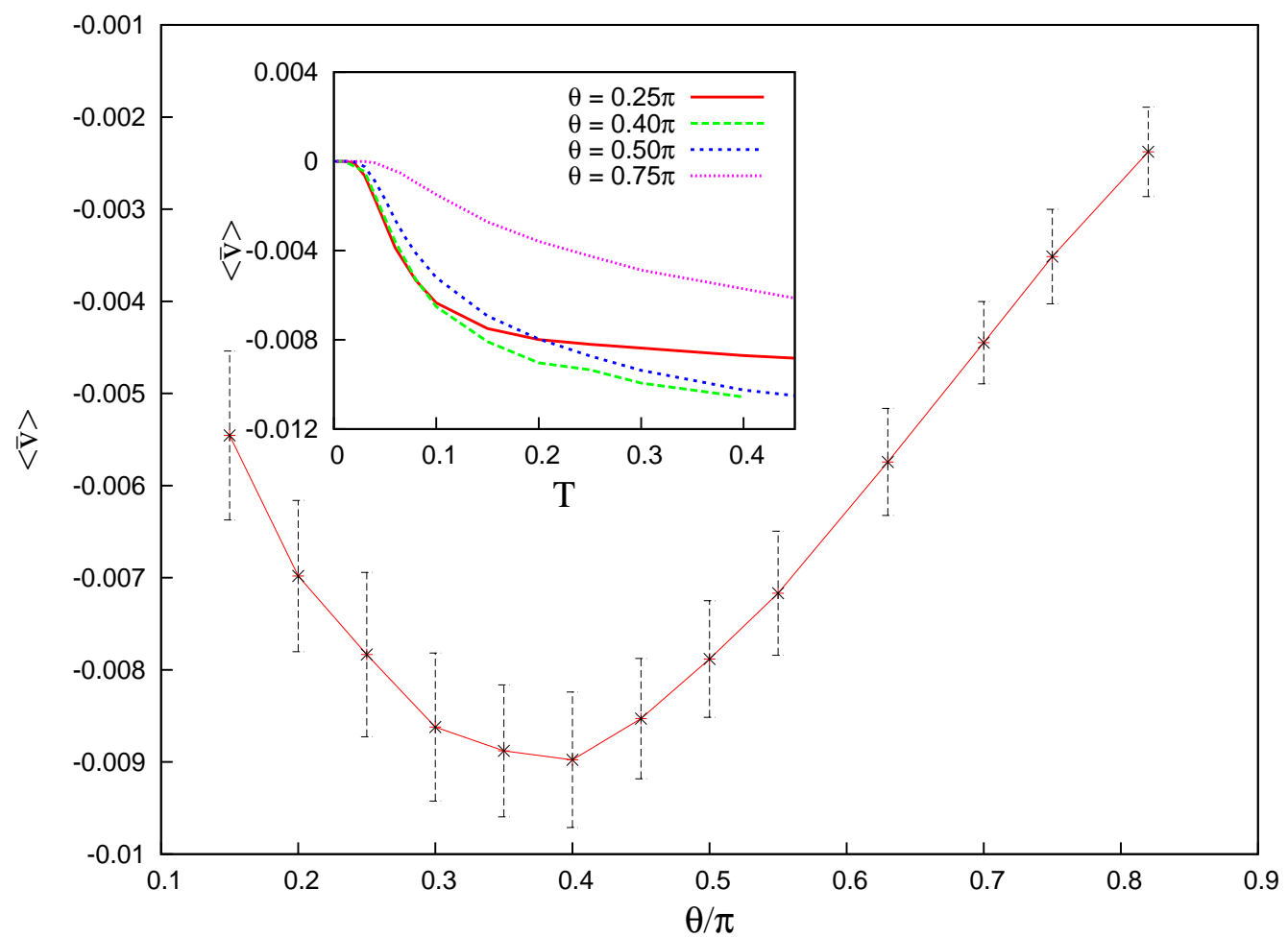

FIG. 10: Variation of $\langle\bar{v}\rangle$ with $\theta$ at $\mathrm{T}=0.2$ for $\tau=8.07, \lambda=0.9$ and $F_{0}=0.2$. The inset shows the variation of $\langle\bar{v}\rangle$ with $\mathrm{T}$ for $\tau=8.07$ but for different $\theta$ values as indicated in the plot keeping other parameters same.

it is $17.5 \%$. In other words, the fractions of the LA states are respectively, $61.5 \%, 75 \%$, and $82.5 \%$ for $\theta=0.75 \pi, 0.5 \pi$, and $0.25 \pi$. Since $\bar{W}$ for LA states are much larger compared to $\bar{W}$ for SA states the weightage average $<\bar{W}>$ decreases with $\theta$ because the fraction of LA states decreases with $\theta$. However, the $\bar{W}$ for LA and SA states are monotonically increasing as a function of $\theta$, Fig.6, implying thereby that $<\bar{W}>$ increases with $\theta$ if the fractions are kept fixed. These two opposing trends of variations of $\langle\bar{W}\rangle$ as a function of $\theta$ leads to the peaking behaviour of $\langle\bar{W}>$ as a function of $\theta$ at low temperatures. However, as the temperature is increased the transitions between the LA and SA states allow SA states to appear where previously only LA states existed and vice versa. This makes the coexistence boundaries of Fig.7 blurred and nonexistent and hence $<\bar{W}>(\theta)$ gets smoothened. Fig.11(b) shows that, at $T=0.2,<\bar{W}>(\theta)$ is monotonic which is to be compared with $\langle\bar{v}\rangle$, shown in Fig.10, showing a peak close to $\theta=\frac{\pi}{2}$ irrespective of the choice of $\left(\gamma_{0}, \tau\right)$ point. Thus, there is no correlation between energy absorption and the ratchet current, at least as far as variation with respect to $\theta$ is concerned. 


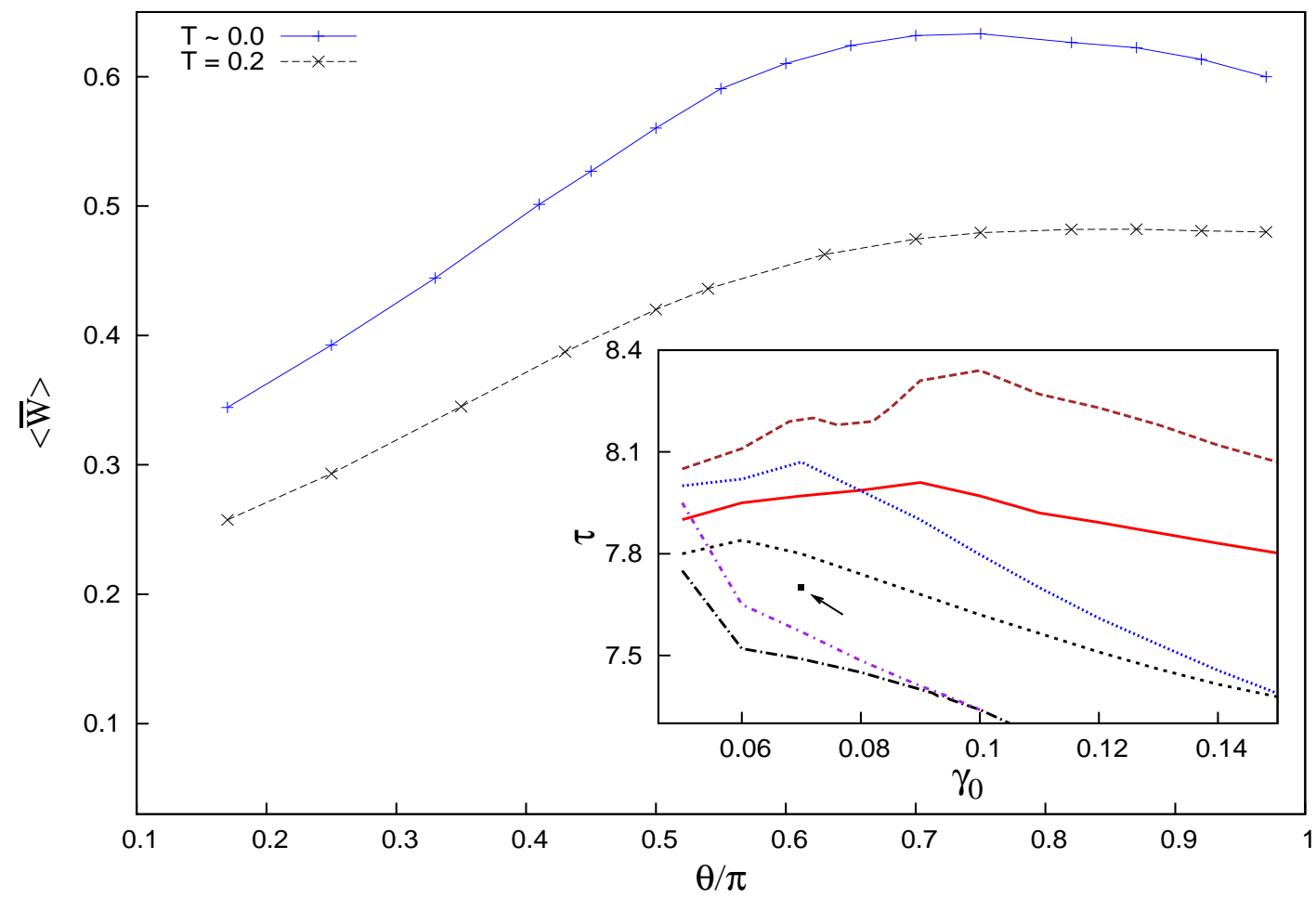

FIG. 11: The variation of $\left\langle\bar{W}>\right.$ as a function of $\theta$ is shown for $\tau=7.70, F_{0}=0.2, \lambda=0.9$, $\gamma_{0}=0.07$ at $T=0.000001$ (blue) and $T=0.2$ (black). The inset shows the loci of constant percentages of population of the SA state in the $(\tau-\gamma)$ space at $T=0.000001$ for various $\theta$ values: $30 \%$ population of SA states when $\theta=0.25 \pi, \theta=0.50 \pi$ and $\theta=0.75 \pi$ are, respectively, depicted by red, black dotted and black dash-dotted lines, whereas for $40 \%$ population of SA states are correspondingly shown in brown, blue and purple. The point $\left(\gamma_{0}=0.07, \tau=7.7\right)$ is marked in the inset.

Figure 7 also provides a guideline to choose the region of $\left(\gamma_{0}, \tau\right)$ space in order to obtain stochastic resonance for a given $\theta$. Invariably, stochastic resonance occurs if the region of $\left(\gamma_{0}, \tau\right)$ space is chosen such that both the dynamical states coexist, at low temperatures (for example, $T=0.000001$ ), with their fractions not too far away from 0.5. In Fig.12, the mean energy absorbed $\left\langle\bar{W}>\right.$ is plotted against temperature for various values of $\tau$ with $\gamma_{0}=0.07$. In Fig.13, the ratchet current $\langle\bar{v}\rangle$ is plotted for the same parameters. From these figures one can observe that stochastic resonance and ratchet effect do occur simultaneously in the same parameter space. Note, however, that the temperatures at which $\langle\bar{W}>$ and $<\bar{v}>$ peak are widely separated; stochastic resonance and peak of ratchet current do not occur at close by temperatures. Similar results have also been obtained earlier when a 


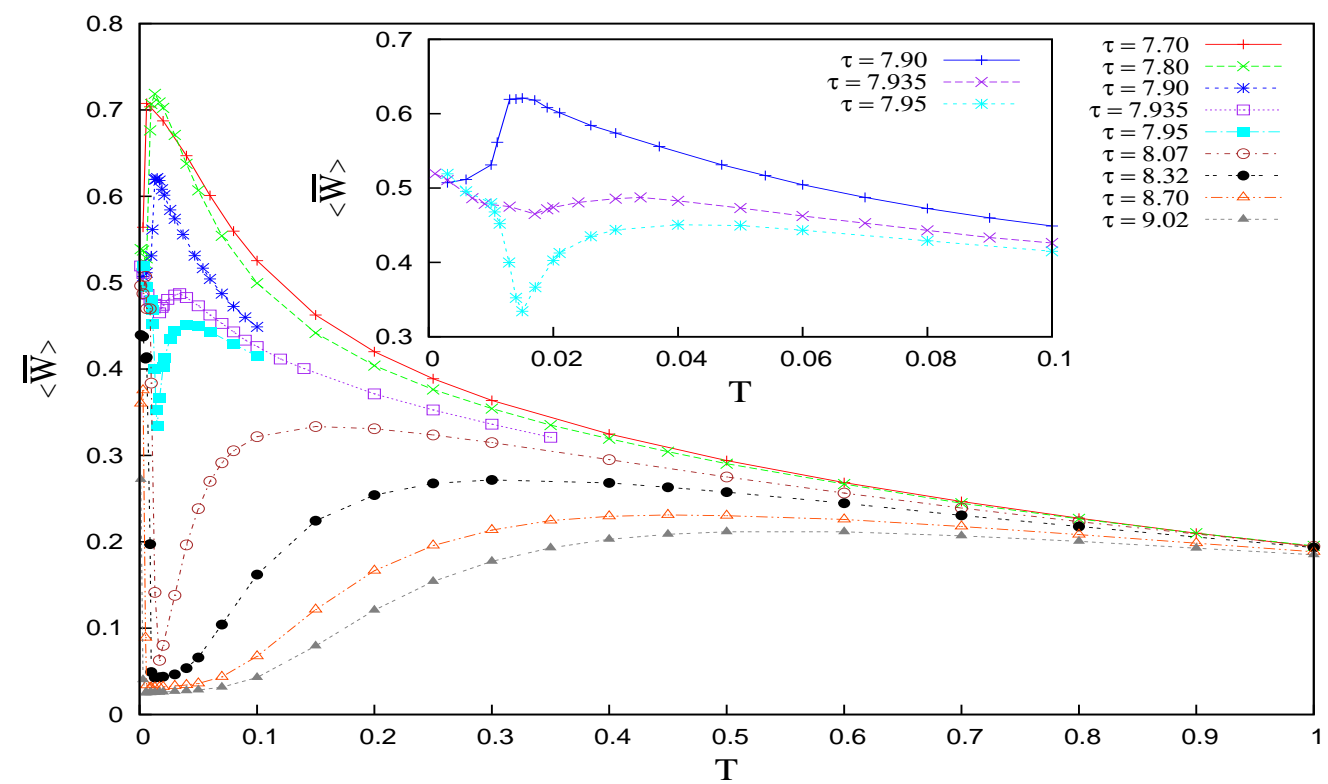

FIG. 12: Variation of $\langle\bar{W}>$ as a function of $T$ for various $\tau$ values as indicated in the top-right corner of the figure with $F_{0}=0.2, \gamma_{0}=0.07, \lambda=0.9, \theta=0.50 \pi$. The inset displays the two differing nature of input energy variations at low temperatures with $\tau \approx 7.935$ as the boundary.

homogeneous system was driven by a biharmonic forcing[26]. Figs. 12 and 13, obtained for an inhomogeneous system driven by a sinusoidal field, are the main results of the present work and deserve further analysis.

Figure 14 shows the variation of the input energy (or energy absorbed) per cycle, $\bar{W}$, of $F(t)$ as a function of the period $\tau$ of $F(t)$ in the LA and SA states at very low temperatures $T \approx 0$ and $\gamma_{0}=0.07$. Of course, the comparison makes sense only in the coexistence region of $\left(\gamma_{0}, \tau\right)$ space. The trend of variation of $\bar{W}$ qualitatively follows that of the amplitude $x_{0}$ of the trajectories, as indicated by the curves in the inset of the figure. The trend remains the same as the temperature is increased by a small amount. Note that in the LA state the system absorbs more energy per cycle than when it is in the state SA. Of course, the mean input energy $\langle\bar{W}>$, as discussed earlier, is the weightage average of $\bar{W}$ in the two states. In view of these results and based on the nature of variation of the curves in Fig. 12 at low temperatures, the set of curves in Fig.12 can be put in two qualitatively distinct groups: one roughly with $\tau \gtrsim 7.935$ and the other with $\tau \lesssim 7.935$, though the dividing line $\tau=7.935$ is not precise. The decrease of $\langle\bar{W}\rangle$ due to thermal fluctuations as the temperature is raised from $T=0$ indicates transition from LA state to SA state. This shows that for the former group of curves with larger $\tau$ the dynamical (SA) state is more stable compared to 


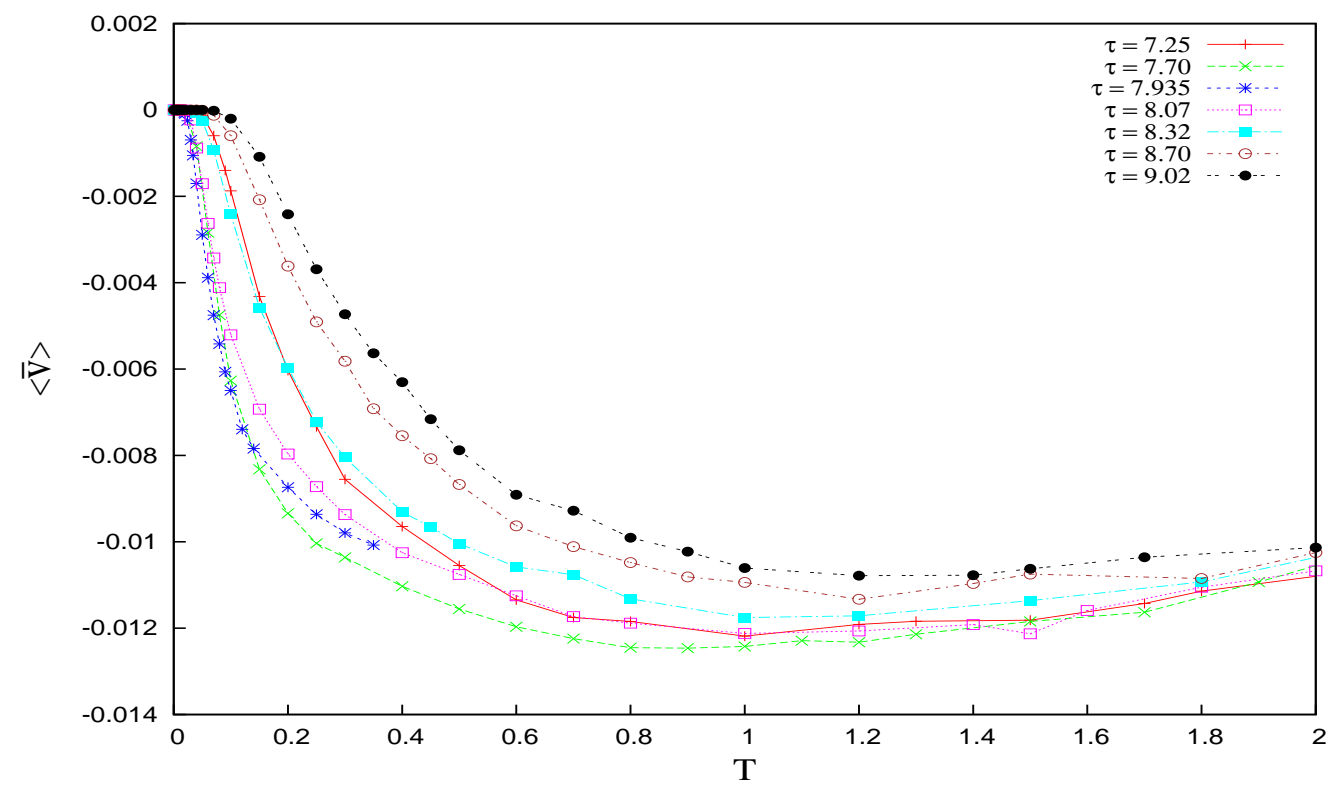

FIG. 13: Variation of $\langle\bar{v}\rangle$ as a function of $T$ for various $\tau$ values as indicated in the top-right corner of the figure with $F_{0}=0.2, \gamma_{0}=0.07, \lambda=0.9, \theta=0.50 \pi$.

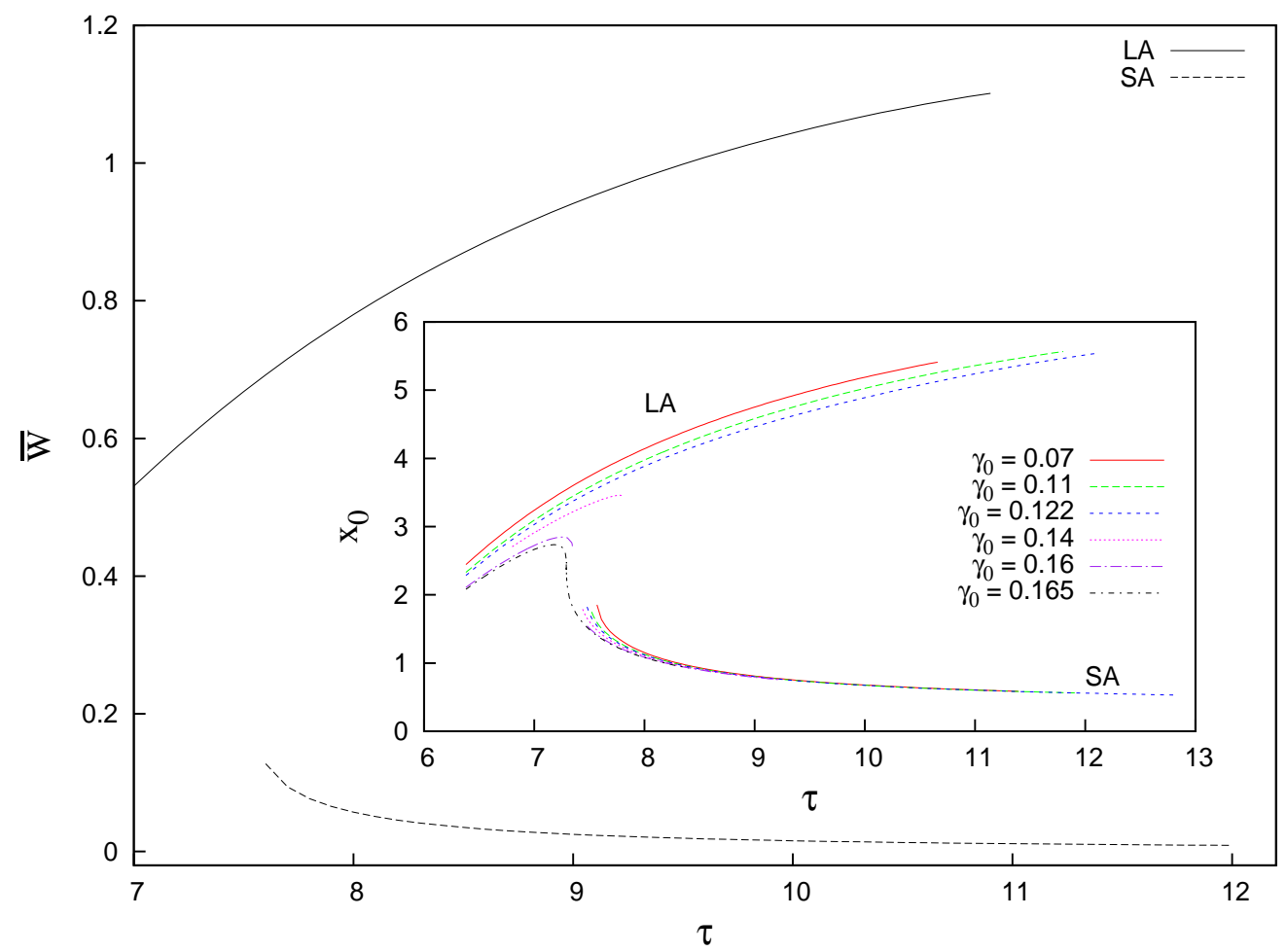

FIG. 14: Variation of $\bar{W}$ as a function of $\tau$ at $T=0.000001$ for the two dynamical (LA and SA) states as indicated, with $F_{0}=0.2, \gamma_{0}=0.07, \lambda=0.9, \theta=0.50 \pi$. The inset shows the variation of amplitude $x_{0}$ as a function of $\tau$ for both LA and SA states at $T=0.000001, \lambda=0.9, F_{0}=0.2, \theta$ $=0.5 \pi$ for different $\gamma_{0}$ values as indicated. 
the LA state. On the other hand, for the latter group of curves with lower $\tau$ the LA state is more stable compared to the SA state. This is consistent with the diagram shown in Fig.7. This is also understandable because larger is the frequency (smaller period) of drive larger should be the phase lag and hence the LA state (with larger phase lag) should be preferred and vice versa.

\section{DISCUSSION AND CONCLUSION}

We presented above the results of our calculations as a function of $T$ and $\theta$ for various values of $\gamma_{0}$ and $\tau$. The results are consistent with each other and bear plausible explanations. However, some detailed results as a function of $\tau$ (or frequency, $\frac{2 \pi}{\tau}$ ) need further scrutiny, which at present we lack clear explanation for.

In Figs. $15 \mathrm{a}$ and $\mathrm{b}$ are presented the variations of $\langle\bar{W}\rangle$ and $\langle\bar{v}\rangle$ as a function of $\tau$ for $\gamma_{0}=0.07$ and $\theta=0.5 \pi$ at various temperatures. Particular attention may be given to the occurrence of maxima of $\langle\bar{W}>$ and $\langle\bar{v}>$ at low temperatures as summarized in Fig.16.

From Fig.14, it is clear that $\bar{W}$ for LA states is much larger than for the SA states, one would, therefore, expect $<\bar{W}>$ to peak just before the SA states make their appearance around $\tau=7.5$, unless the loss due to appearance of SA states is more than compensated for by the rise of $\bar{W}$ with increase in $\tau$. However, the slope of $\bar{W}(\tau)$ is not so large as to offset the loss due to appearance of the SA states, yet $<\bar{W}>$ peaks at a value of $\tau$ larger than 7.5. Though this anomaly is curious, the peaking of $\langle\bar{v}\rangle$ at low temperatures is more interesting. $\langle\bar{v}>$ peaks at a value of $\tau \sim 7.935$ where SA and LA states are nearly equally stable. (As concluded earlier, at smaller $\tau$ the LA state is more stable than SA state

and vice versa.) In other words, at low temperatures, the asymmetry in the probability of crossings to the left and right wells of the periodic potential becomes the largest at that value $\tau$ for which LA and SA states are equally stable!

Though $\langle\bar{W}>$ and $\langle\bar{v}>$ do not peak at the same $\tau$ values, the difference in $\tau$ values at which they peak is much smaller compared to the range of $\tau$ over which the two states coexist. However, for given values of $\gamma_{0}$ and $\tau,\langle\bar{W}>$ and $\langle\bar{v}>$ peak at widely separated temperatures, Fig.16. Thus the variation of $\langle\bar{W}\rangle$ and $\langle\bar{v}\rangle$ as a function of frequency is nearer to coincidence than the peaking of $\langle\bar{W}>$ (stochastic resonance) and $<\bar{v}>$ as a 

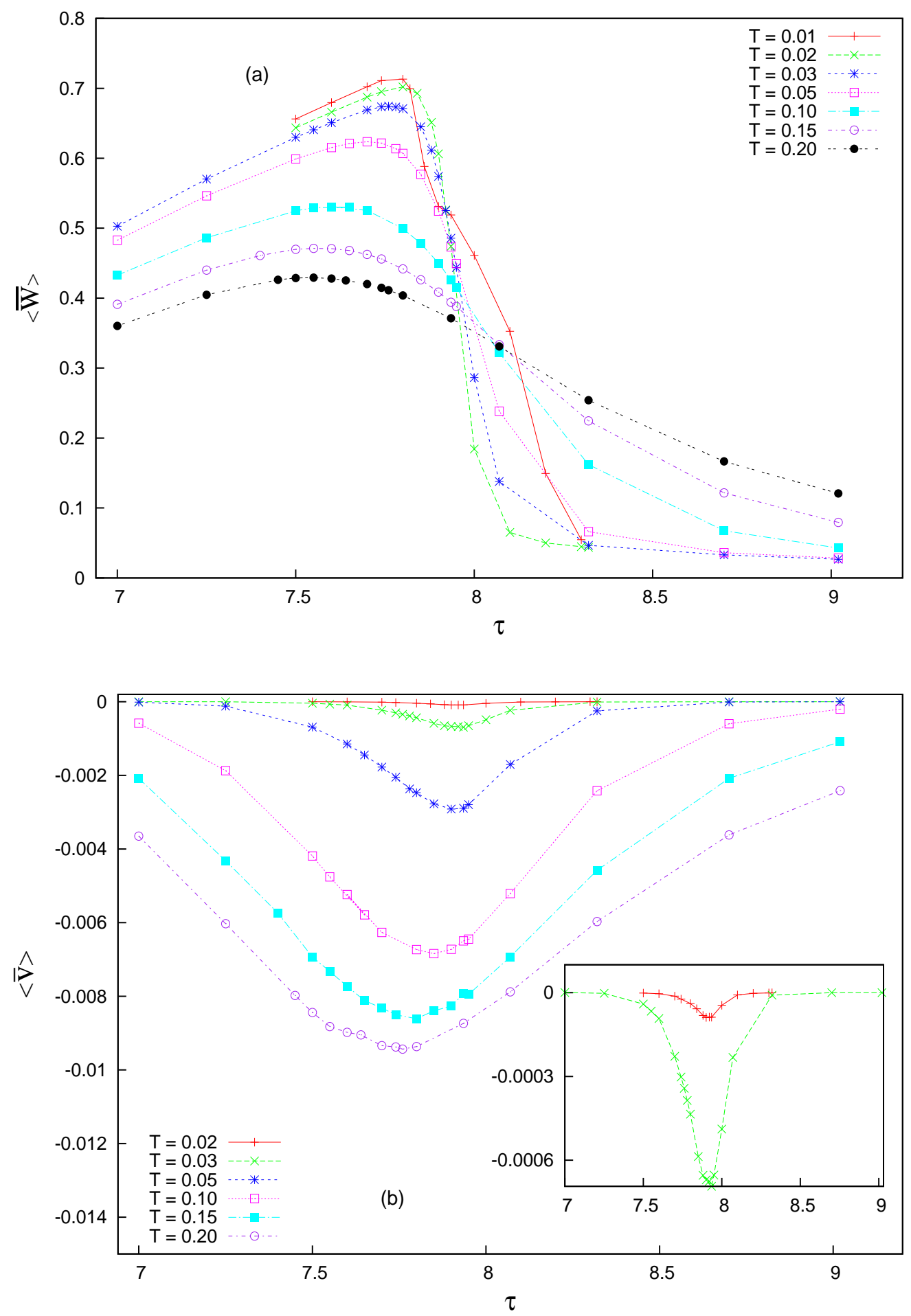

FIG. 15: Variation of $\langle\bar{W}>$ as a function of $\tau$ is shown in fig.15(a) and that of $\langle\bar{v}>$ in fig.15(b) for various small temperatures as indicated, with $F_{0}=0.2, \gamma_{0}=0.07, \lambda=0.9, \theta=0.50 \pi$. The inset in Fig.15(b), with the same axes label as the main plot, shows the two curves for lower temperatures $T=0.02$ and $T=0.03$ for clarity. 


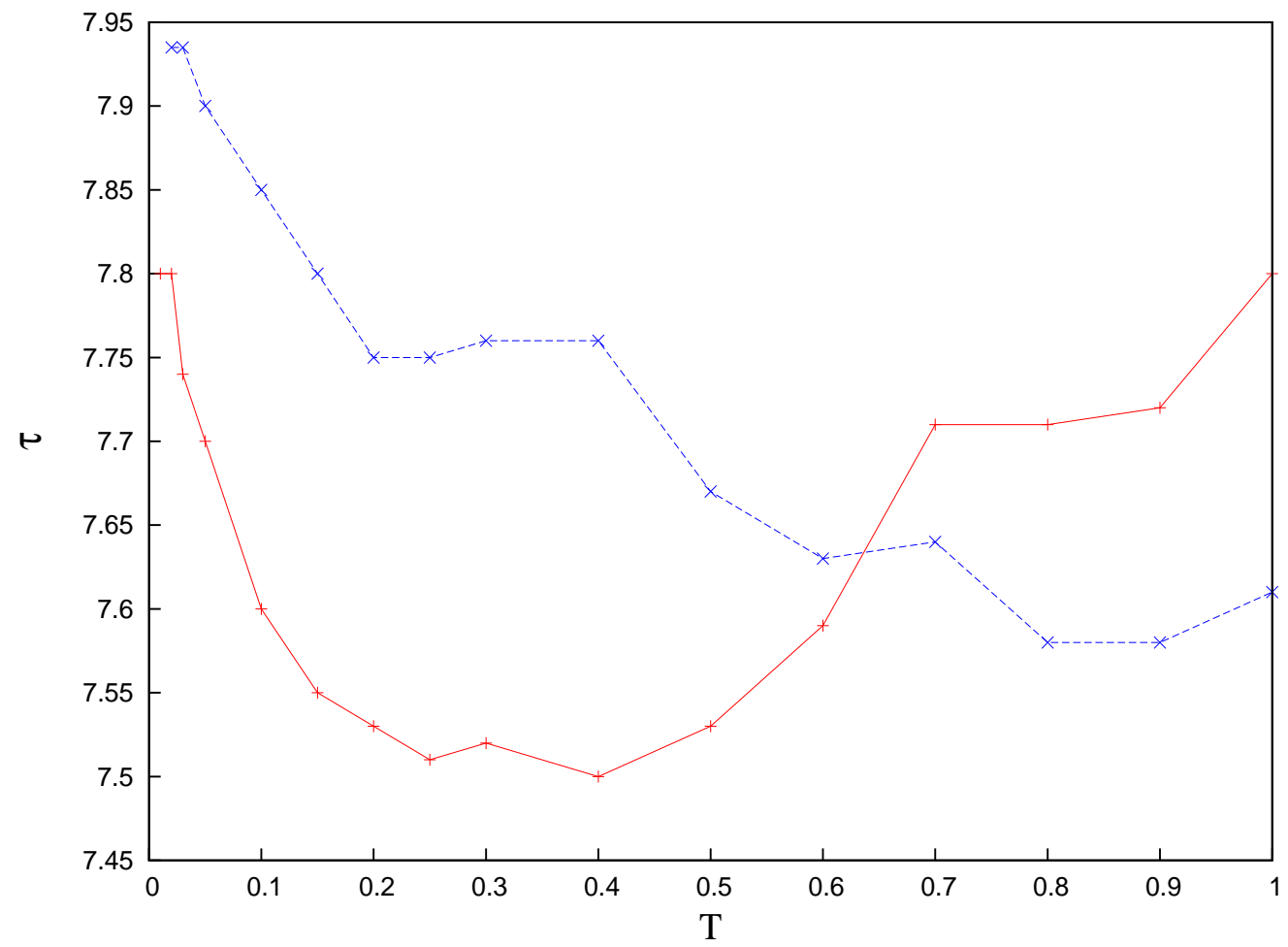

FIG. 16: The figure records the $\tau$ values corresponding to maximum $\langle\bar{W}>$ (in red) and maximum $<\bar{v}>$ (in blue) as $T$ is changed, with $F_{0}=0.2, \gamma_{0}=0.07, \lambda=0.9, \theta=0.50 \pi$.

function of temperature.

As stated earlier, frictional inhomogeneity is a comparatively weak agent to yield ratchet effect, yet the maximum ratchet current that we obtain is not negligible and there is a possibility of obtaining still larger current if the parameters are tuned suitably. For example, we have not yet completely explored the variation of $\left\langle\bar{W}>\right.$ and $\left\langle\bar{v}>\right.$ as a function of $\gamma_{0}$ which may yield larger ratchet current.

In summary, we are closer to finding answer to all questions that we had mentioned to begin with. We could observe stochastic resonance and ratchet effect in the same region of parameter space. However, SR and ratchet current peak do not occur at the same temperature. We now have a better idea about the relative roles played by the parameters $\theta$ and $\lambda$ characterising the frictional inhomogeneity and also the periodicity $\tau$ of the external periodic field to obtain $\langle\bar{W}>$ and $\langle\bar{v}>$.

We thank the Computer Centre, North-Eastern Hill University, Shillong, for providing 
the high performance computing facility, SULEKOR.

[1] H. Risken, The Fokker-Planck Equation Ch. 11, Springer-Verlag, 1989.

[2] K. Svoboda, C.F. Schmidt, B.J. Schnapp, and S.M. Block, Nature 365, 721 (1993); J.T. Finer, R.S. Simmons, and J.A. Spudich, Nature 368, 113 (1994). 694,1 94

[3] P. Reimann, Phys. Rep. 361, 57 (2002).

[4] M.O. Magnasco, Phys. Rev. Lett. 71, 1477 (1993); ibid 72, 2656 (1994).

[5] J. Prost, J. F. Chauwin, L. Peliti, and A. Ajdari, Phys. Rev. Lett. 72, 2652 (1994); R.D. Astumian, and M.Bier, Phys. Rev. Lett. 72, 1766 (1994).

[6] F. Jülicher, A. Ajdari, and J. Prost, Rev. Mod. Phys. 69, 1269 (1996).

[7] J. Maddox, Nature 369, 181 (1994); ibid 369, 271 (1994).

[8] J. Rousselet, L. Salome, A. Ajdari, and J. Prost, Nature 370, 446 (1994).

[9] R. Benzi, A. Sutera, and A. Vulpiani J. Phys. A 14, L453 (1981).

[10] L. Gammaitoni, P. Hänggi, P. Jung, and F. Marchesoni, Rev. Mod. Phys. 70, 223 (1998).

[11] T. Wellens, V. Shatokhin, and A. Buchleitner, Rep. Prog. Phys. 67, 45 (2004).

[12] B. McNamara, and K. Wiesenfeld, Phys. Rev. A 39, 4854 (1989).

[13] S. Fauve, and F. Heslot, Phys. Lett. A 97, 5 (1983).

[14] B. McNamara, K. Wiesenfeld, and R. Roy, Phys. Rev. Lett. 60, 2626 (1988).

[15] S. Saikia, A.M. Jayannavar, and M.C. Mahato, Phys. Rev. E 83, 061121 (2011); W.L. Reenbohn, S.S. Pohlong, and M.C. Mahato, Phys. Rev. E 85, 031144 (2012); S. Saikia, Physica A 46, $411(2014)$

[16] W.L. Reenbohn, and M.C. Mahato, Phys. Rev. E 88, 032143 (2013).

[17] K. Liu, and Y. Jin, Physica A 392, 5283 (2013).

[18] Y.W. Kim, and W. Sung, Phys. Rev. E 57, R6237 (1998).

[19] M. Büttiker, Z. Phys. B - Condensed Matter, 68, 161 (1987).

[20] R. Landauer, J. Stat. Phys. 52, 233 (1988).

[21] Ya M. Blanter, and M. Büttiker, Phys. Rev. Lett. 81, 4040 (1998).

[22] R. Benjamin, and R. Kawai, Phys. Rev. E 77, 051132 (2008).

[23] W.L. Reenbohn, and M.C. Mahato, J. Stat. Mech.:Theory and Experiment P03011 (2009).

[24] D. Dan, M.C. Mahato, and A.M. Jayannavar, Phys. Rev. E 60, 6421 (1999); D. Dan, A.M. 
Jayannavar, and M.C. Mahato, Int. J. Mod. Phys. 14,1585 (2000); D. Dan, M.C. Mahato, and A.M. Jayannavar, Physica A 296, 375 (2001); D. Dan, M.C. Mahato, and A.M. Jayannavar, Phys. Rev. E 63, 056307 (2001); W.L. Reenbohn, S. Saikia, R. Roy, and M.C. Mahato, Pramana - Journal of Physics, 71, 297 (2008).

[25] M. Qian, Y. Wang, and X-J. Zhang, Cin. Phys. Lett. 20, 810 (2003).

[26] W.L. Reenbohn, and M.C. Mahato, Phys. Rev. E 91, 052151 (2015).

[27] J.M. Sancho, M. San Miguel, and D. Duerr, J. Stat. Phys. 28, 291 (1982).

[28] A.M. Jayannavar, and M.C. Mahato, Pramana - Journal of Physics, 45, 369 (1995).

[29] E.A. Desloge, Am. J. Phys. 62, 601 (1994).

[30] W.H. Press, B.P. Flannery, S.A. Teukolsky, and W.T. Vetterling, Numerical Recipes, Cambridge University Press, Cambridge, England (1987).

[31] M.C. Mahato, and S.R. Shenoy, Phys. Rev. E 50, 2503 (1994); T. Iwai, Physica A 300, 350 (2001); M. Evstigneev, P. Reimann, C. Schmitt, and C.Bechinger, J. Phys.: Condens. Matter 17, S3795 (2005).

[32] R. Mannella, A Gentle Introduction to the Integration of Stochastic Differential Equations. In : Stochastic Processes in Physics, Chemistry, and Biology. Edited by J. A. Freund and T. Pöschel, Lecture Notes in Physics, vol. 557, 353. Springer, Berlin, 2000.

[33] K. Sekimoto, J. Phys. Soc. Jpn. 66, 1234 (1997). 